\title{
On the characterization of tripled partial metrics and tripled quasi-metrics
}

\author{
Ghorban Khalilzadeh Ranjbar * • Mohammad Esmael \\ Samei
}

Received: 23.10.2019 / Revised: 28.03.2019 / Accepted: 13.04.2020

\begin{abstract}
The purpose of this study is to define a tripled partial metric, quasi-metric spaces, and present the relationship between the notion of tripled partial metric, which has applications in computer sciences, that of quasi-metric and that of standard metric. We give some simple formulations of the sequences convergence and of the completeness in tripled partial metric space.
\end{abstract}

Keywords. Partial metric, tripled partial metric, tripled quasi-metric, weighted.

Mathematics Subject Classification (2010): 2010 Mathematics Subject Classification: 54E35, 54H25, $47 \mathrm{H} 10$

\section{Introduction}

The notion of partial metric belongs to Matthews [8,9], being aimed as a generalization of the usual metric spaces $X$ in which the distance $d(x, x)$ is not necessarily zero for $x \in X$. Many researcher worked in $S$-metric space and describe some fixed point theorems quasimetrics spaces $([7,2-4,10,12,5])$. Matthews provided also a partial metric version of the Banach fixed point theorem. His study was related to denotational semantics of data flow networks, and is still applied to models in the theory of computation. There is a strong relationship between partial metrics and quasi-metrics, partially noted already by Matthews. A quasi-metric is not symmetric, but it satisfies the triangle inequality ([16]). In section 3, we present the notions of tripled partial metric and weighted quasi-metric and we provide the characterization of tripled partial metrics in terms of usual metrics and non-expansive functions, and then in terms of weighted quasi-metrics with non-expansive weights. A characterization of weighted quasi-metrics in terms of metrics is also given. The new element here is the non-expansiveness of the functions which appear in the characterization of the tripled partial metrics and quasi-metrics.

\footnotetext{
* Corresponding author
}

G. Khalilzadeh Ranjbar

Department of Mathematics, Faculty of Science, Bu-Ali Sina University, Hamedan 65178, Iran

E-mail: gh_khalilzadeh@yahoo.com

M.E. Samei

Department of Mathematics, Azarbaijan Shahid Madani University, Tabriz, Iran

E-mail: mesamei@gmail.com 


\section{Preleminaries}

We begin by giving the definitions of partial metrics and quasi metric.

Definition 2.1 Let $X$ be a nonempty set. A partial metric is a function $p: X \times X \rightarrow[0, \infty)$ satisfying, for all $x, y, z \in X$, the following conditions:

$\left(p_{1}\right) x=y$ if and only if $p(x, x)=p(x, y)=p(y, y)$;

$\left(p_{2}\right) p(x, x) \leq p(x, y)$;

$\left(p_{3}\right) p(x, y)=p(y, x)$ (symmetric);

(p $\left.p_{4}\right) p(x, y) \leq p(x, z)+p(z, y)-p(z, z)$.

Remark 2.1 The condition $\left(p_{4}\right)$ generalizes the triangle inequality of a metric.

Definition 2.2 Let $X$ be a nonvoid set. A quasimetric is a function $q: X \times X \rightarrow[0, \infty)$ satisfying, for all $x, y, z \in X$, the following conditions

$\left(q_{1}\right) x=y$ if and only if $q(x, y)=q(y, x)=0$;

$\left(q_{2}\right) q(x, y) \leq q(x, z)+q(z, y)$. If $q$ is a quasimetric, then $d: X \times X \rightarrow[0, \infty)$ given by

$$
d(x, y)=\frac{q(x, y)+q(y, x)}{2}
$$

is a metric named the associated metric to $q$.

Matthews ([8,9]) defined also the notion of weighted quasimetric.

Definition 2.3 Let $X$ be a nonvoid set. A weighted quasimetric is a quasimetric $q: X \times$ $X \rightarrow[0, \infty)$ for which there exists a function $\alpha: X \rightarrow[0, \infty)$, called weight, so that

$$
q(x, y)+\alpha(x)=q(y, x)+\alpha(y)
$$

for all $x, y \in X$.

To each partial metric, one can associate a usual metric in a natural way.

Theorem 2.1 ([8]) If $p$ is a partial metric, the function $d: X \times X \rightarrow[0, \infty)$ given by

$$
d(x, y)=p(x, y)-\frac{p(x, x)+p(y, y)}{2}
$$

for all $x, y \in X$, is a metric on $X$, called the associated metric to $p$.

\section{Main results}

We begin by giving the deffinitions of tripled partial metric and tripled quasimetric.

Definition 3.1 Let $X$ be a nonempty set. A tripled partial metric is a function $p: X \times X \times$ $X \rightarrow[0, \infty)$ which defines as follows:

$\left(p_{1}\right) p(x, y, z)=p(x, z, y)=p(y, x, z)=p(z, y, x)$ for all $x, y, z \in X$;

( $\left.p_{2}\right) x=y=z$ if and only if $p(x, x, x)=p(y, y, y)=p(z, z, z)=p(x, y, z)$;

( $\left.p_{3}\right) p(x, x, x), p(y, y, y), p(z, z, z) \leq p(x, y, z)$ for all $x, y, z \in X$;

$\left(p_{4}\right) p(x, y, z) \leq p(x, y, a)+p(x, a, z+p(a, y, z)-p(a, a, a)$ for all $x, y, z, a \in X$.

Example 3.1 Let $X=\{0,1,4\}$ be endowed with the partial metric $p: X \times X \times X \rightarrow$ $[0, \infty)$ defined by

$$
p(x, y, z)=\frac{1}{4}|x-y|+\frac{1}{4}|x-z|+\frac{1}{4}|y-z|+\frac{1}{2} \max \{x, y, z\} .
$$

$p(1,1,1)=\frac{1}{2} \neq 0, p(4,4,4)=2 \neq 0$. So $p$ is not a metric on $X$. 
Definition 3.2 If $p$ is a tripled partial metric on $X$, then $q: X \times X \times X \rightarrow[0, \infty)$ defined by $q(x, y, z)=p(x, y, z)-p(x, x, x)$ is a tripled quasimetric.

Definition 3.3 An open $p$-ball for $x \in X, \varepsilon>0$ is defined by

$$
\begin{aligned}
B(x, x, \varepsilon) & =\{(y, z) \in X \times X \mid q(x, y, z)<\varepsilon\} \\
& =\{(y, z) \in X \times X p(x, y, z)<q(x, x, x)+\varepsilon\} .
\end{aligned}
$$

Remark 3.1 Each tripled partial metric $p$ on $X$ generates a $t_{0}$-topology on $X$ which has a base the family open $p$-balls.

Definition 3.4 A metric d can be defined by a tripled partial metric $p$ as follows

$$
d(x, y, z)=\max \{q(x, y, z), q(y, x, z), q(z, y, x)\}
$$

where $q$ is tripled quasimetric.

Definition 3.5 Tripled metric, on $X$ is a function $d: X \times X \times X \rightarrow[0, \infty)$, such that defines as follows

$\left(d_{1}\right) d(x, y, z)>0$ if and only if $x \neq y$ or $y \neq z$ or $x \neq z$ or $x \neq y \neq z$;

$\left(d_{2}\right) d(x, y, z)=0$ if and only if $x=y=z$;

$\left(d_{3}\right) d(x, y, z)=d(z, x, y)=d(y, x, z)=d(x, z, y)$;

$\left(d_{4}\right) d(x, y, z) \leq d(x, y, a)+d(a, y, z)+d(x, a, z)$ for all $x, y, z, a \in X$.

Example $3.2 d: \mathbb{R}^{3} \rightarrow \mathbb{R}$ as follows

$$
d(x, y, z)=|x-y|+|y-z|+|x-z| .
$$

Definition 3.6 If $q$ is tripled quasimetric, then

$$
d(x, y, z)=\frac{q(x, y, z)+q(z, y, x)+q(y, x, z)}{3}
$$

is called a tripled associated metric to $q$.

Definition 3.7 Let $X$ be a nonvoid set. A weighted tripled quasimetric is a quasimetric $q: X \times X \times X \rightarrow[0, \infty)$ for which there exists a function $\alpha: X \rightarrow[0, \infty)$, called weight so that

$$
q(x, y, z)+\alpha(x)=q(y, x, z)+\alpha(y)=q(z, y, x)+\alpha(z)
$$

for all $x, y, z, \in X$.

Definition 3.8 If $p$ is a tripled parial metric, the function $d: X \times X \times X \rightarrow[0, \infty)$ given by

$$
d(x, y, z)=p(x, y, z)-\frac{p(x, x, x)+p(y, y, y)+p(z, z, z)}{3}
$$

is a metric on $X$ called the associated metric to $p$.

Definition 3.9 The function $\varphi: X \rightarrow[0, \infty)$ is called non-expansive respect to $d$ if $[2 \varphi(x)-(\varphi(y)+\varphi(z))] \leq d(x, y, z)$ or $[2 \varphi(y)-(\varphi(x)+\varphi(z))] \leq d(y, x, z)$, also $[2 \varphi(z)-(\varphi(x)+\varphi(y))] \leq d(z, x, y)$ for all $x, y, z \in X$.

We give characterization of tripled partial metrics in terms of tripled metric and nonexpansive functions. 
Theorem 3.1 A function $p: X \times X \times X \rightarrow[0, \infty)$ is a tripled partial metric on $X$ if and only if there exists a metric $d$ and a non-expansive function $\varphi: X \rightarrow[0, \infty)$ with respect to $d$, so that

$$
p(x, y, z)=d(x, y, z)+\varphi(x)+\varphi(y)+\varphi(z)
$$

for all $x, y, z \in X$. Furthermore $d$ and $\varphi$ are uniquely determined by $p$.

Proof. Let $p$ be a tripled partial metric and given $d: X \times X \times X \rightarrow[0, \infty)$ by

$$
d(x, y, z)=p(x, y, z)-\frac{p(x, x, x)+p(y, y, y)+p(z, z, z)}{3},
$$

$\varphi(x)=\frac{p(x, x, x)}{3}$, we get

$$
3 \varphi(x)=p(x, x, x) \leq p(x, y, z)=d(x, y, z)+\varphi(x)+\varphi(y)+\varphi(z) .
$$

Then we have $2 \varphi(x) \leq d(x, y, z)+\varphi(y)+\varphi(z)$, indeed

$$
2 \varphi(x)-(\varphi(y)+\varphi(z)) \leq d(x, y, z),
$$

and we can obtain $2 \varphi(y)-(\varphi(x)+\varphi(z))<d(x, y, z)$ and

$$
2 \varphi(z)-(\varphi(x)+\varphi(y)) \leq d(x, y, z) .
$$

Conversely, given a metric $d$ and a non-expansive function $\varphi$, we have to prove that $p$ given by $p(x, y, z)=d(x, y, z)+\varphi(x)+\varphi(y)+\varphi(z)$ satisfies the conditions $\left(p_{1}\right),\left(p_{2}\right),\left(p_{3}\right)$ and $\left(p_{4}\right)$.

Con. $\left(p_{1}\right)$ : for all $x, y, z \in X, p(x, y, z)=p(x, z, y)=p(y, x, z)=p(z, y, x)$. It is obvious.

Con. $\left(p_{2}\right): x=y=z$ if and only if

$$
p(x,, x, x)=p(y, y, y)=p(z, z, z)=p(x, y, z) .
$$

We have $p(x, x, x)=d(x, x, x)+3 \varphi(x)=3 \varphi(x)$. Conversely, if $d(x, y, z)=0$ then $x=y=z$.

Con. $\left(p_{3}\right): p(y, y, y) \leq p(y, x, z), p(y, z, x)$. Since

$$
d(y, y, y)+3 \varphi(y) \leq d(y, x, z)+\varphi(y)+\varphi(x)+\varphi(z),
$$

$2 \varphi(y)-(\varphi(x)+\varphi(z)) \leq d(y, x, z)$.

Con. $\left(p_{4}\right): p(x, y, z) \leq p(x, y, a)+p(x, a, z)+p(a, y, z)-p(a, a, a)$, Hence

$$
\begin{aligned}
d(x, y, z)+\varphi(x)+\varphi(y)+\varphi(z) \leq & d(x, y, a)+\varphi(x)+\varphi(y)+\varphi(a) \\
& +d(x, a, z)+\varphi(x)+\varphi(a)+\varphi(z) \\
& +d(a, y, z)+\varphi(a)+\varphi(y)+\varphi(z)-p(a, a, a) .
\end{aligned}
$$

We give a characterization of tripled quasimetric in terms of tripled metric and of tripled partial metric.

Theorem 3.2 Let $q$ is quasimetric. The following assertions are equivalent

(a) $q$ is weighted;

(b) there exist a metric $d$ and a function $\varphi: X \rightarrow[0, \infty)$ so that the relation $q(x, y, z)=$ $d(x, y, z)-2 \varphi(x)+\varphi(y)+\varphi(z)$ holds;

(c) there exists a function $\psi: X \rightarrow[0, \infty)$ so that $(x, y, z) \rightarrow q(x, y, z)+3 \varphi(x)$ is a partial metric. 
Furthermore,

(1) $d$ is a unique (and equal the associated metric to q),

(2) $\varphi$ is a unique up to on additive constant,

(3) $\psi=\varphi$ is constant.

Proof. (a) $\Rightarrow$ (b): Let $d$ be the associated metric to $q$ given by

$$
d(x, y, z)=\frac{q(x, y, z)+q(y, x, z)+q(z, y, x)}{3} .
$$

If $\alpha$ is a weight for $q$, then we have

$$
\begin{aligned}
d(x, y, z)-2 \varphi(x)+\varphi(y)+\varphi(z)= & \frac{q(x, y, z)+q(y, x, z)+q(z, y, x)}{3} \\
& -2 \varphi(x)+\varphi(y)+\varphi(z) .
\end{aligned}
$$

If $\varphi(x)=\frac{\alpha(x)}{3}$, we get

$$
\begin{aligned}
q(x, y, z)+\alpha(x)= & q(y, x, z)+\alpha(y) \\
= & q(z, y, x)+\alpha(z) \\
= & \frac{1}{3}[q(x, y, z)+q(y, x, z)+\alpha(x)-\alpha(y) \\
& +q(z, y, x)+\alpha(x)-\alpha(z)] \\
& -2 \frac{\alpha(x)}{3}+\frac{\alpha(y)}{3}+\frac{\alpha(z)}{3} \\
= & q(x, y, z)+\frac{2 \alpha(x)}{3}-\frac{\alpha(y)}{3}-\frac{\alpha(z)}{3} \\
& -\frac{2 \alpha(x)}{3}+\frac{\alpha(y)}{3}+\frac{\alpha(z)}{3} .
\end{aligned}
$$

(b) $\Rightarrow$ (c): Take $\psi=\varphi$. We have

$$
q(x, y, z)=d(x, y, z)-2 \varphi(x)+\varphi(y)+\varphi(z),
$$

hence

$$
2 \varphi(x)-(\varphi(y)+\varphi(z)) \leq d(x, y, z) .
$$

Notice that the function $\varphi$ is $d$ nonexpansive. We have

$$
q(x, y, z)+3 \varphi(x)=d(x, y, z)+\varphi(y)+\varphi(z)+\varphi(x)
$$

and this is a partial metric.

(c) $\Rightarrow\left(\right.$ a): There exist metric $d$ and a $d$-nonexpansive function $\left.\left.\varphi_{1}: X \rightarrow\right] 0, \infty\right)$ such that

$$
q(x, y, z)+3 \psi(x)=d(x, y, z)+\varphi_{1}(x)+\varphi_{1}(y)+\varphi_{1}(z) .
$$

Taking here $x=y=z$, We obtain $\varphi_{1}(x)=\psi(x)$. Hence

$$
\begin{aligned}
q(x, y, z)+3 \psi(x) & =d(x, y, z)+\psi(x)+\psi(y)+\psi(z) \\
d(x, y, z) & =\frac{q(x, y, z)+q(y, x, z)+q(z, y, x)}{3} .
\end{aligned}
$$


We get

$$
\begin{aligned}
& q(x, y, z)+2 \psi(x)=d(x, y, z)+\psi(y)+\psi(z) \\
& q(y, x, z)+2 \psi(y)=d(y, x, z)+\psi(x)+\psi(z) \\
& q(z, y, x)+2 \psi(z)=d(z, y, x)+\psi(y)+\psi(x) .
\end{aligned}
$$

Thus

$$
\begin{gathered}
q(x, y, z)+q(y, x, z)+q(z, y, x)+2 \psi(x)+2 \psi(y)+2 \psi(z) \\
=3 d(x, y, z)+2 \psi(z)+2 \psi(x)+2 \psi(y),
\end{gathered}
$$

so $d$ is the associated metric to $q$. The uniqueness of $d$ it follows from the fact that $q(x, y, z)+$ $q(y, x, z)+q(z, y, x)=3 d(x, y, z)$, for a fixed $x_{0} \in X$, we obtain

$$
\begin{aligned}
q\left(x, x_{0}, x_{0}\right) & =d\left(x, x_{0}, x_{0}\right)-2 \varphi(x)+\varphi\left(x_{0}\right)+\varphi\left(x_{0}\right), \\
2 \varphi(x) & =d\left(x, x_{0}, x_{0}\right)-q\left(x, x_{0}, x_{0}\right)+2 \varphi\left(x_{0}\right) .
\end{aligned}
$$

Hence the uniqueness of $\varphi$ up to an additive follows. Using this fact and

$$
q(x, y, z)=d(x, y, z)-2 \varphi(x)+\varphi(y)+\varphi(z)
$$

one obtains that $\psi=\varphi$ is constant.

Corollary 3.1 The function $q: X \times X \times X \rightarrow[0, \infty)$ is a weighted quasimetric if and only if there exists a metric $d$ and a d-non-expansive function $\varphi: X \rightarrow[0, \infty)$ so that $q(x, y, z)=d(x, y, z)-2 \varphi(x)+\varphi(y)+\varphi(z)$.

Example 3.3 Let $X=\{[a, b] \mid a \leq b\}$. We define $\varphi([a, b])=\frac{b-a}{3}, \alpha([a, b])=b-a$,

$$
\begin{aligned}
d([a, b],[c, d],[e, f])= & \frac{1}{2}(|a-c|+|b-d|+|a-e| \\
& +|b-f|+|c-e|+|d-f|)
\end{aligned}
$$

and

$$
\begin{aligned}
p([a, b],[c, d],[e, f])= & \max \{b, d\}-\min \{a, c\}+\max \{b, f\} \\
& -\min \{a, e\}+\max \{f, d\}-\min \{c, e\} .
\end{aligned}
$$

Example 3.4 Let

$$
C=\left\{F: \mathbb{N} \rightarrow(0, \infty) \mid \sum_{n=0}^{\infty} 2^{-n} \frac{1}{F(n)}<\infty\right\} .
$$

The function $p: C \times C \times C \rightarrow[0, \infty)$ defined by

$$
\begin{aligned}
p(F, G . H)= & \sum_{n=0}^{\infty} 2^{-n}\left[\max \left\{\frac{1}{F(n)}, \frac{1}{G(n)}\right\}\right. \\
& \left.+\max \left\{\frac{1}{G(n)}, \frac{1}{H(n)}\right\}+\max \left\{\frac{1}{F(n)}, \frac{1}{H(n)}\right\}\right],
\end{aligned}
$$

is a partial metric. We define $\varphi(F)=\frac{1}{3} \sum_{n=0}^{\infty} 2^{-n} \frac{1}{F(n)}$ and so

$$
d(F, G, H)=\frac{1}{2} \sum_{n=0}^{\infty} 2^{-n}\left(\left|\frac{1}{F(n)}-\frac{1}{G(n)}\right|+\left|\frac{1}{G(n)}-\frac{1}{H(n)}\right|+\left|\frac{1}{H(n)}-\frac{1}{F(n)}\right|\right)
$$


is tripled metric. Thus

$$
\left\{\begin{array}{l}
q: C \times C \times C \rightarrow[0, \infty) \\
q(F, G . H)=\sum_{n=0}^{\infty} 2^{-n}\left\{\left(\frac{1}{G(n)}-\frac{1}{F(n)}\right)^{+}\right. \\
\left.\quad+\left(\frac{1}{H(n)}-\frac{1}{G(n)}\right)^{+}+\left(\frac{1}{H(n)}-\frac{1}{F(n)}\right)^{+}\right\}
\end{array}\right.
$$

is a weighted quasimetric with the weight $\alpha(F)=\sum_{n=0}^{\infty} 2^{-n} \frac{1}{F(n)}$, where $x^{+}=\max \{x, 0\}$.

Definition 3.10 In a tripled partial metric space $(X, p), x_{n} \stackrel{p}{\rightarrow} x$ if and only if

$$
\lim _{n \rightarrow \infty} \sup \left(d\left(x_{n}, x, x\right)+\varphi\left(x_{n}\right)-\varphi(x)\right) \leq 0 .
$$

Definition 3.11 The Cauchy sequence $\left\{x_{n}\right\}$ are characterized by

$$
\lim _{m, n \rightarrow \infty} d\left(x_{n}, x_{m}, x_{m}\right)=0
$$

and $\lim _{n \rightarrow \infty} \varphi\left(x_{n}\right)=0$.

Definition 3.12 $(X, p)$ is complete if for each Cauchy sequence $\left\{x_{n}\right\}$ there exist $x \in X$ such that $\varphi(x)=0$ and $\lim _{n \rightarrow \infty} d\left(x_{n}, x, x\right)=0$.

Theorem 3.3 Let $(X, q)$ be a weighted quasimetric space and denote by d, the associated metric. The following assertions are equivalent

1) $X$ is complete with respect to $d$.

2) Each $f: X \rightarrow X$ for which there exists a function $\Phi: X \rightarrow[0, \infty)$ lower semicontinuous with respect to do such that

$$
q(x, x, f(x)) \leq \Phi(x)-\Phi(f(x))
$$

for all $x \in X$, has a fixed point on $X$.

Proof. From Corollary 3.1, we get

$$
q(x, y, z)=d(x, y, z)-2 \varphi(x)+\varphi(y)+\varphi(z) .
$$

$\varphi$ being nonexpansive and the condition (3.1) we have

$$
\begin{aligned}
q(x, x, f(x) & =d(x, x, f(x))-2 \varphi(x)+\varphi(x)+\varphi(f(x)) \\
& \leq \Phi(x)-\Phi(f(x)) \\
d(x, x, f(x)-\varphi(x)+\varphi(f(x)) & \leq \Phi(x)-\Phi(f(x)) \\
d(x, x, f(x)) & \leq \varphi(x)-\varphi(f(x))+\Phi(x)-\Phi(f(x)) \\
& =(\varphi+\Phi)(x)-(\varphi+\Phi)(f(x)) .
\end{aligned}
$$

By applying the standard Caristi theorems for the lower semi-continuous function $\varphi+\Phi$, we obtain the conclusion. For the converse, suppose that $(X, d)$ is not complete. Denoting by $(\tilde{X}, \tilde{d})$ it completion, there exists a sequence $\left\{a_{n}\right\}$ in $X$ such that $a_{n} \rightarrow a_{\infty}$ as $n \rightarrow \infty$, where $a \in \tilde{X}-X$. For $n \in \mathbb{N}, x \in X$

$$
\begin{aligned}
q\left(x, x, a_{n}\right) & =d\left(x, x, a_{n}\right)-2 \varphi(x)+\varphi(x)+\varphi\left(a_{n}\right) \\
& \leq 2 d\left(x, x, a_{n}\right) \rightarrow 2 \tilde{d}(x, x, a)>0
\end{aligned}
$$


as $n \rightarrow \infty$. Then

$$
3 \tilde{d}\left(x, x, a_{\infty}\right)-3 \tilde{d}\left(a_{n}, a_{n}, a_{\infty}\right)>2 \tilde{d}\left(x, x, a_{\infty}\right) .
$$

It follow that there exists $n \in \mathbb{N}$ such that

$$
q\left(x, x, a_{n}\right)<3 \tilde{d}\left(x, x, a_{\infty}\right)-3 \tilde{d}\left(a_{n}, a_{n}, a_{\infty}\right) .
$$

Denote by $n(x)$ the smallest $n$ for which this inequality holds. Defining $f: X \rightarrow X$ by $f(x)=\alpha_{n(x)}$ and $\Phi: X \rightarrow[0, \infty)$ by $\Phi(x)=3 \tilde{d}\left(x, x, a_{\infty}\right)$ one obtain that $q(x, x, f(x))<$ $\Phi(x)-\Phi(f(x))$. The function $\Phi$ is obviously continuous and $f$ has no fixed point, contradiction.

\section{References}

1. Acar, O., Altun, I., Romaguera, S.: Caristi's type mappings on complete partial metric spaces, Fixed Point Theory, 14 (1), 3-10 (2013).

2. Anisiu, M.C., Anisiu, V., Kása, Z.: Total palindrome complexity of finite words, Discrete Mathematics, 310, 109-114 (2011). doi:10.1016/j.disc.2009.08.002

3. Caristi, J.: Fixed point theorems for mappings satisfying inwardness conditions, Transactions of the american mathematical society, 215, 241-251 (1976). doi: 10.1090/S0002-9947-1976-0394329-4

4. Cobzaş, S.: Completeness in quasi-metric spaces and Ekeland variational principle, Topology and its Applications, 158, 1073-1084 (2011). doi:10.1016/j.topol.2011.03.003

5. Ding, H.S., Ozturk, V., Radenović, S.: On some new fixed point results in b-rectangular metric spaces, Journal of Nonlinear Sciences and Applications, 8, 378-386 (2015). doi: 10.22436/jnsa.008.04.10

6. Haghi, R.H., Rezapour, Sh., Shahzad, N.: Be careful on partial metric fixed point results, Topology and its Applications, 160 (3), 450-454 (2013). doi: 10.1016/j.topol.2012.11.004

7. Khalilzadeh Ranjbar, G., Samei, M.E.: A generalization of fixed point theorems about $F$-contraction in particular $S$-metric spaces and characterization of quasi-contraction maps in b-metric spaces, University Politehnica of Bucharest Scientific Bulletin, Series A, 81 (2), 51-64 (2019).

8. Matthews, S.G.: Partial metric spaces, 8th British Colloquium for Theoretical Computer Science, (1992).

9. Matthews, S.G.: Partial metric topology, In: Proceedings of the 8th Summer Conference on General Topology and Applications, Annals of the New York Academy of Sciences, 728, 183-197 (1994).

10. Mustafa, Z., Parvaneh, V., Jaradat, M., Kadelburg, Z.: Extended rectangular b-metric spaces and some fixed point theorems for contractive mappings, Symmetry, 11 (4), 594 (2019). doi: 10.3390/sym11040594

11. Romaguera, S., Kirk, A.: Type characterization of completeness for partial metric spaces, Fixed Point Theory and Applications, 2010, Article ID.493298, 6 page (2010). doi: 10.1155/2010/493298

12. Roshan, J.R., Parvaneh, V., Altun, I.: Some coincidence point results in ordered b-metric spaces and applications in a system of integral equations, Applied Mathematics and Computation, 226, 725-737 (2014). doi: 10.1016/j.amc.2013.10.043

13. Samet, B.: Existence and uniqueness of solutions to a system of functional equations and applications to partial metric spaces, Fixed Point Theory, 14 (2), 473-481 (2013). 
14. Schellekens, M.: The Smyth completion: a common foundation for denotational semantics and complexity analysis, Electronic Notes in Theoretical Computer Science, 1, 535-556 (1995). doi: 10.1016/S1571-0661(04)00029-5

15. Turinici, M.: Function contractive maps in partial metric spaces, Journal of Mathematics, 2013, Article ID 637521, 6 pages (2013). doi: 10.1155/2013/637521

16. Wilson, W.A.: On quasi-metric spaces, American Journal of Mathematics, 53 (3), 675684 (1931). doi: 10.2307/2371174 